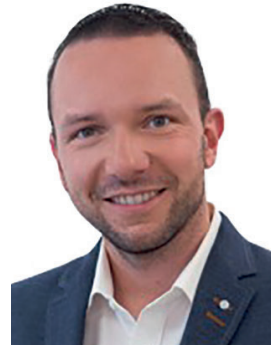

Mario Mader

Koordination «DermaCampus»

\section{Von Rezepturen und Vorbereitungen}

\begin{abstract}
Trotz des ständig steigenden Angebotes an Fertigarzneimitteln haben Rezepturen ihren Stellenwert in der Dermatologie nicht verloren. Da aber dem Management und Monitoring topischer Therapien inklusive der Rezepturen weder während des Medizinstudiums noch während der Facharztzeit ausreichend Beachtung geschenkt wird, stellt Prof. Dr. Petra Staubach-Renz - Mitglied der Fachgruppe Magistralrezepturen der Gesellschaft für Dermopharmazie (DG) und Sonderreferentin für Rezepturen des Berufsverbands der Deutschen Dermatologen (BVDD) - in dieser Ausgabe die wichtigsten Aspekte zur Verordnung von Rezepturen mit hoher pharmakologischer und medizinischer Qualität vor.

Eine Schwangerschaft beeinflusst nicht nur das Privatleben, sondern auch das Arbeitsleben. Oft ist diese Veränderung begleitet von viel Unsicherheit, sowohl auf Seiten der werdenden Mutter als auch auf Seiten des Arbeitgebers. Gerade bei Ärztinnen sind aber einige spezielle Punkte zu berücksichtigen, um den besonderen Schutz für Mutter und Kind zu gewährleisten. Der Deutsche Ärztinnenbund stellt eine Übersicht mit Tipps und Hinweisen zur Verfügung, die kurz und knapp erläutert, was die betroffenen Ärztinnen, aber auch Arbeitgeber oder Kollegen und Kolleginnen beachten sollten.
\end{abstract}

Magistralrezepturen im Zeitalter der personalisierten Medizin Wo stehen wir 2019?

\section{Einleitung}

Das Fach der dermatologisch tätigen Ärzte hat sich in den letzten Jahren stark verändert. Obwohl die systemischen Therapien immer mehr im Vordergrund stehen, bleibt die Lokaltherapie inklusive der Rezepturen die Domäne der Dermatologie. Das Fertigarzneimittelspektrum hat sich erweitert, trotzdem gibt es viele therapeutische Nischen, die mit Rezepturen optimal besetzt werden. Im Zeitalter der evidenzbasierten Medizin wird die Verordnung von Rezepturen/Magistralrezepturen diskutiert; in der personalisierten Medizin insbesondere bei seltenen Indikationen, besonderen Bedingungen von Inhaltsstoffen und Erkrankungen sowie Neuerungen im therapeutischen Spektrum sind Rezepturen bevorzugt Magistralrezepturen unverzichtbar.

\section{Curriculum Magistralrezepturen}

Das Management und Monitoring topischer Therapien inklusive der Rezepturen wird weder während des Medizinstudiums noch während der Facharztzeit ausreichend Beachtung geschenkt. Ne- ben der Physiologie und Pathophysiologie der Haut sollte man gerade im 21. Jahrhundert auch ein Grundwissen zu den Wirkstoffen, Vehikelsystemen, Basistherapeutika sowie Hintergrund zur Plausibilitätsprüfung, die durch Apotheken durchzuführen sind, haben. Als mögliche Verordnungsoptionen, die die Patientenversorgung besonders in der topischen Therapie optimieren, entsteht gerade ein Curriculum Magistralrezepturen, wo diese Grundkenntnisse zum Verordnen von Rezepturen mit hoher pharmakologischer und medizinischer Qualität vermittelt werden. Eine neue Leitlinie zum richtigen Einsatz von topischen Therapien ist im letzten Jahr entstanden [1]. Weiterhin gibt es auf der Homepage der Gesellschaft für Dermopharmazie eine Fachgruppe für Magistralrezepturen, die in regelmäßigen Abständen ihre Rezepturenleitlinie und Wirkstoffdossiers für dermatologische Rezepturen überarbeiten.

Fakt ist: Trotz des ständig steigenden Angebotes an Fertigarzneimitteln haben Rezepturen ihren Stellenwert in der Dermatologie nicht verloren. 3 von 10 Verordnungen durch Dermatologen sind Rezepturen. Individualrezepturen stehen immer noch im Vorder-

\section{KARGER}

Fax +497614520714

information@karger.com

www.karger.com
() 2019 S. Karger GmbH, Freiburg

Accessible online at: www.karger.com/kkd 
grund, obwohl die Qualität der Rezeptur durch den verstärkten Einsatz von Magistralrezepturen verbessert werden könnte.

\section{Was ist der Unterschied zwischen Magistral- und} Indiviualrezeptur?

Magistralrezepturen sind Rezepturen, die auf der Grundlage standardisierter und geprüfter (Rezeptur-)Vorschriften hergestellt wird, in Veröffentlichungen zu finden ist und bereits auf Plausibilität überprüft wurde. Inkompatibilitäten, Wechselwirkungen, Wirkstoffqualität und -quantität sind überprüft. Die Individualrezeptur dagegen sind individuelle, meist überlieferte Rezepturen, deren Plausibilität nicht überprüft wurde.

Hilfestellungen zur Verordnung von Rezepturen: Wo finde ich geeignete Quellen und Tipps zu Magistralrezepturen?

- Neues Rezeptur-Formularium (NRF) - Standardisierte Formelsammlung für Ärzte

Dies ist ein Sammelwerk mit unzähligen Magistralrezepturen, davon mehr als 200 für dermatologische Indikationen. Eine komprimierte Version für die Kitteltasche ist erhältlich und bietet bei Fragen auch die Möglichkeit der Kommunikation über die NRFHotline (siehe Abb.).

- Hautarzt: Rezepturtipps für die Praxis aus der Praxis

- Formelsammlungen der Arzneimittel- oder Rohstoffhersteller mit auf Plausibilität geprüften Rezepturen

- Rezeptur-Hotlines von Arznei- und Rohstoffherstellern sowie NRF

\section{Was mache ich, wenn ich auf meine Individualrezeptur nicht} verzichten will?

Rezeptur-Hotlines von Rohstofflieferanten oder das NRF bieten hier Hilfe. Sie können die Rezeptur entweder auf Plausibilität prüfen oder abklären, ob eine adäquate Magistralrezeptur mit vergleichbarer Zusammensetzung bereits in Formelsammlungen verfügbar ist. Die Zusammenarbeit mit einem benachbarten oder bekannten Apotheker ist dabei hilfreich. Alternativ kann beim BVDD die Sonderreferentin für Rezepturen (Autorin dieses Artikels) angeschrieben werden, die dann die geeignete Vernetzung auf kurzen Wegen einleiten kann.

\section{Indikationen für Magistralrezepturen 2019 - Therapeutische}

Lücken

(1) Variation der Arzneistoffkonzentration/Wirkklasse/Grundlage Wirkstoffe in verschiedenen Konzentrationen in jedem Lebensalter, z.B. 0,08-0,15\% für Säuglinge und Kleinkinder oder im Alter (NRF 11.144).

(2) Moderne Kortikosteroide in zinkhaltigen Grundlagen für die intertriginösen Bereiche

Prednicarbat 0,08-0,25\% oder Mometasonfuroat 0,1\% in Hydrophilem Zinkoxid-Liniment 25\% (NRF 11.109).

(3) Basispflege bei entzündlichen chronischen Dermatosen Die Bedeutung der Basispflege wird immer deutlicher. Ein monatlicher Verbrauch von einem Kilogramm ist bei optimaler Anordnung bekannt. Bei Kindern bis zum 12. Lebensjahr dürfen diese Therapeutika verordnet werden, wenn die Qualität der Inhaltsstoffe einer Arzneibuchqualität entspricht. Hier können hilfreich

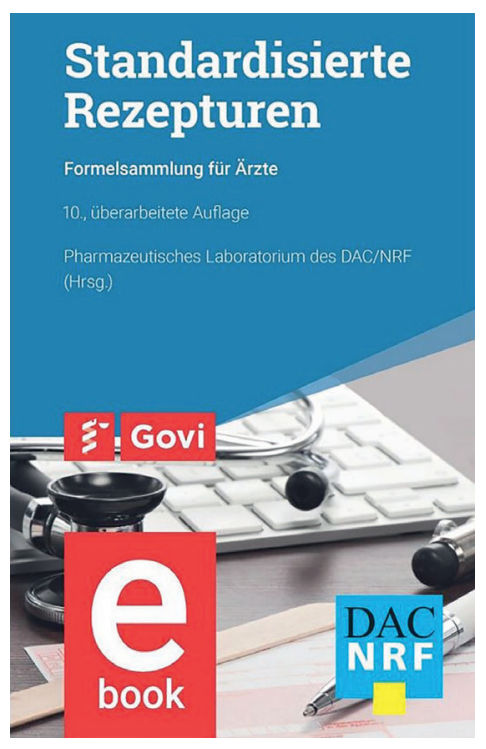

Das neue Rezeptur-Formularium im Kitteltaschenformat [2].

Rezepturen eingesetzt werden, z.B. Kühlcreme DAB, Hydrophile Harnstoff-Emulsion 5\%/10\% (NRF 11.72), Lipophile Harnstoff-Natriumchlorid-Salbe (NRF 11.75). Wirkstoff wenig stabil oder als Fertigarzneimittel nur bedingt verfügbar, z.B. Dithranol- oder Capsaicinhaltige Externa. Abwaschbare Dithranol-Salbe 0,05-2\% mit Salicylsäure 2\% (NRF 11.52). Kombination mehrerer Arzneistoffe. Prednicarbat 0,08-0,25\%, Octenidindihydrochlorid 0,1\% Creme (NRF 11.145), Salicylsäure-Öl 2-10\% mit Triamcinolonacetonid 0,1\% (NRF 11.134), Triamcinolonacetonid-Creme 0,025-0,1\% mit Chlorhexidindigluconat 1\% (NRF 11.136).

(4) Vermeidung potenter Allergene

Verzicht bzw. Austausch von Hilfs- und Konservierungsstoffen.

(5) Altbewährte Rezepturen

Gerade im Zeitalter der multiresistenten Keime können zinkoder ammoniumbituminosulfonathaltige Rezepturen als Antiseptika eingesetzt werden.

(6) Beeindruckende Therapie (Adährenzerhöhung durch personalisierte Therapie)

Die Adhärenz ist in der Dermatologie von besonderer Bedeutung. Das Schlagwort der personalisierten Therapie kann auch für die Rezepturenindikation herangezogen werden.

(7) Verordnungsmenge beliebig variierbar

Verordnungsmengen (Richtgröße ab $200 \mathrm{~g}$ ) sind als Magistralrezeptur in der Regel kostengünstiger (Stichwort: Krankenhausapotheken involvieren).

\section{Zusammenfassung}

Dermatologische Magistralrezepturen sind sinnvoll und haben ihren Stellenwert nicht nur in der Dermatologie. Durch die Zusammenarbeit zwischen Dermatologen und Pharmazeuten können therapeutische Lücken mit kurzen Wegen überwunden werden. Durch eine verbesserte Adhärenz und kostengünstigeres Verordnen bei Großmengen sind die sozioökonomischen Aspekte nicht zu vernachlässigen. Leitlinien, Rezepturhotlines sowie das NRF (auch im Kitteltaschenformat) unterstützen hier in der täglichen Praxis. 


\section{Weiterführender Link und Literatur}

1 www.awmf.org/leitlinien/detail/1l/013-092.html

2 Pharmazeutisches Laboratorium des DAC/NRF (Hrsg): Standardisierte Rezepturen. Formelsammlung für Ärzte, ed 10. Eschborn, Avoxa - Mediengruppe Deutscher Apotheker. 2018.
Prof. Dr. Petra Staubach-Renz ist Oberärztin ᄂ

und Poliklinik der Universitätsmedizin Mainz und Nıı Fachgruppe Magistralrezepturen der Gesellschaft für Dermopharmazie (GD) sowie Sonderreferent für Rezepturen des Berrufsverbands der Deutschen Dermatologen (BVDD).

\section{Arbeitsrecht \\ Das kann die ÄRZTIN tun ... \\ $\square$ Impfstatus überprüfen \\ $\square$ Informationen über das Mutterschutzgesetz, die Schwanger- schaft und die üblichen Abläufe in der Abteilung bzw. Praxis einholen \\ 『 Fahrplan erstellen (z.B. Zeitpunkt für offizielles Bekanntgeben der Schwangerschaft festlegen, wie kann ich meine bisherige eigenverantwortliche Arbeit als Fach- oder Oberärztin weiter- führen) \\ 『 Gespräch mit der/dem Vorgesetzten suchen \\ 『 Termin für die Gefährdungsbeurteilung \\ 『 Verbündete unter den Kolleginnen und Kollegen suchen und sich kollegial zeigen (z.B. Sonn- und Feiertagsdienste und -schichten bis 22 Uhr übernehmen - natürlich nur, wenn da- bei Alleinarbeit ausgeschlossen ist) \\ 『 betriebsärztliche Erhebung des Immunstatus}

Operieren in der Schwangerschaft - Tipps und Hinweise für die Praxis vom Deutschen Årztinnenbund*

Fragen für das Gespräch mit der/dem Vorgesetzten ... kann ich noch was vor dem Mutterschutz durchführen?

? Wie kann ich auf der Intensivstation arbeiten?

? Welche Hausbesuche kann ich weiterhin machen? und organisatorische Tätigkeiten auszuüben? schutz und ggf. Elternzeit für mich?

\section{Das kann der ARBEITGEBER tun ...}

$\square$ Schwangerschaften grundsätzlich als normal ansehen und den Kompetenzzuwachs der Frau im Blick haben

『 jeder Schwangeren für alle erkennbar den Rücken stärken und sie mit den nötigen Informationen versorgen

$\square$ ein Beschäftigungsverbot nur als Ultima Ratio aussprechen

$\square$ in der Praxis dafür sorgen, dass die Schwangere neue Patienten «vorsortiert» zugeteilt bekommt (z.B. keine Patienten mit infektiösen Erkrankungen)

$\square$ kein primäres Interesse an der Rückerstattung der «Ausfallpauschale»

$\square$ fachliche Weiterbildung und die weitere Karriere während und nach dem Mutterschutz bzw. der Elternzeit gemeinsam mit der Ärztin planen

$\square$ die Furcht der werdenden Mutter vor einer Fruchtschädigung am Arbeitsplatz respektieren und sinnvolle alternative Beschäftigungsmöglichkeiten erörtern

$\square$ die gesetzlich vorgeschriebene Gefährdungsbeurteilung eines jeden Arbeitsplatzes voranzutreiben, nicht erst, wenn eine Frau schwanger wird

$\square$ in Fortbildungen und per Aushang die Reform des Mutterschaftsrechts thematisieren

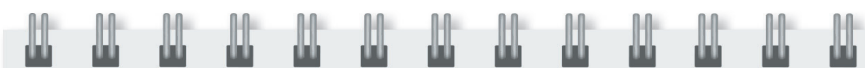

? Wiekann ich weiterhin operativ oder interventionell tätig sein?

? Folgendes fehlt mir noch für meinen Weiterbildungskatalog,

? Welche Möglichkeiten gibt es, verstärkt wissenschaftliche

? Welche beruflichen Perspektiven gibt es hier nach Mutter-

Das ist bei operativer oder interventioneller Tätigkeit zu tun..

! Betreuung elektiver, nachweislich nicht infektiöser Patienten «Notfälle sind tabu»

! Besonderes Augenmerk auf den Eigenschutz legen (z.B. doppelte Handschuhe, Schutzbrille)

! Patient vor jeder Operation untersuchen, ob eine nicht impfbare Infektion vorliegt

! Zeitlimit bei Operationen beachten (4 Stunden)

! Bereitstellen einer Sitzgelegenheit

! Verlassen des Kontrollbereichs bei Röntgenuntersuchungen, wöchentliches Auslesen des Dosimeters mit Zweitdosimeter in Uterushöhe

! BeiTätigkeitin der Anästhesie: nurintravenöse Narkosen (TIVA) anwenden und auf Maskennarkosen und Lachgas verzichten

! Keine Beteiligung an Lagerungsmaßnahmen

! Abgeben der Operation muss jederzeit möglich sein

! Kein Kontakt mit Formalin

*Auszugsweise gestatteter gekürzter Nachdruck des vom Deutschen Ärztinnenbund herausgegebenen Faltblatts «Tipps und Hinweise für die Praxis - Was kann eine Ärztin mit Kinderwunsch tun, wie können Arbeitgeberinnen und Arbeitgeber handeln?» www.aerztinnenbund.de/downloads/5/Kinderwunsch.pdf

\section{Weitere Informationen erteilt}

Dr. med. Barbara Schmeiser, Vizepräsidentin des Deutschen Ärztinnenbundes e.V. (DÄB),

E-Mail: barbara.schmeiser@aerztinnenbund.de

Deutscher Ärztinnenbund 